\title{
Some Relatively New Techniques for Nonlinear Problems
}

\author{
Syed Tauseef Mohyud-Din, ${ }^{1}$ Muhammad Aslam Noor, ${ }^{2}$ \\ and Khalida Inayat Noor ${ }^{2}$ \\ ${ }^{1}$ Department of Basic Sciences, Heavy Industries Taxila Education City (HITEC) University, \\ Taxila Cantt 44000, Pakistan \\ ${ }^{2}$ Department of Mathematics, COMSATS Institute of Information Technology, Islamabad 44000, Pakistan
}

Correspondence should be addressed to Syed Tauseef Mohyud-Din, syedtauseefs@hotmail.com

Received 11 January 2009; Revised 14 February 2009; Accepted 25 March 2009

Recommended by Ji Huan He

This paper outlines a detailed study of some relatively new techniques which are originated by He for solving diversified nonlinear problems of physical nature. In particular, we will focus on the variational iteration method (VIM) and its modifications, the homotopy perturbation method (HPM), the parameter expansion method, and exp-function method. These relatively new but very reliable techniques proved useful for solving a wide class of nonlinear problems and are capable to cope with the versatility of the physical problems. Several examples are given to reconfirm the efficiency of these algorithms. Some open problems are also suggested for future research work.

Copyright (C) 2009 Syed Tauseef Mohyud-Din et al. This is an open access article distributed under the Creative Commons Attribution License, which permits unrestricted use, distribution, and reproduction in any medium, provided the original work is properly cited.

\section{Introduction}

With the rapid development of nonlinear sciences, many analytical and numerical techniques have been developed by various scientists. Most of the developed techniques have their limitations like limited convergence, divergent results, linearization, discretization, unrealistic assumptions and noncompatibility with the versatility of physical problems [1-100]. He [16-40] developed a number of efficient and reliable techniques for solving a wide class of nonlinear problems. These relatively new but very powerful methods proved to be fully synchronized with the complexities of the physical problems, see $[1-7,11-40,49-80,84-100]$ and the references therein. In the present study, we will focus our attention on He's variational iteration (VIM), homotopy perturbation (HPM), modified variational iteration (MVIMS), parameter expansion, and exp-function methods. The variational iteration method (VIM) was suggested and proposed by $\mathrm{He}$ [17-24] in its preliminary form in 1999. The method has been used to solve nonlinear differential 
equations. In a subsequent work [23, 24] the VIM was developed into a full theory for solving diversified physical problems of versatile nature. It is to be highlighted that the variational iteration method (VIM) is also very effective for solving differential-difference equations, see $[49,95]$ and the references therein. Moreover, He [17, 18, 27-38] introduced another wonderful technique, namely, homotopy perturbation (HPM) by merging the standard homotopy and perturbation. The HPM is independent of the drawbacks of the coupled techniques and absorbs all their positive features. It is to be noted that homotopy perturbation is a kind of perturbation method which can take full advantage of various perturbation methods, while using the homotopy technique to guarantee simple solution procedure. It is to worth mentioning that if the initial solution is suitably chosen, then only one or two iterations are enough to get the appropriate result, see [17, 18, 27-38]. The expfunction method was first proposed by $\mathrm{He}$ and $\mathrm{Wu}$ [39] in 2006. The method was originally suggested to search for solitary solutions and periodic solutions of nonlinear wave equations. It always leads to a generalized solution with free parameters which can be determined by using the initial/boundary conditions. The most interesting part is transformation between periodic and solitary solutions by using the so-called He-Wu transformations. The method $[17,39,40,84-87,97,100]$ is always used as a tool to find exact solutions, but it can be utilized also for finding solutions approximately including the solutions for boundary value problems. It is to be highlighted that the present study would also outline the He parameter-expansion technique [18, 25-27]. The parameter expansion technique includes the modified Lindstedt-Pioncare and book keeping parameter methods and previously called the parameter-expanding method. He [18] in his review article in 2006 also explained that the method does not require to construct a homotopy. These efficient techniques have been applied to a wide class of nonlinear problems, see $[1-7,11-40,49-80,84-$ $100]$ and the references therein. With the passage of time some modifications in $\mathrm{He}^{\prime} \mathrm{s}$ variational iteration method (VIM) has been introduced by various authors. Abbasbandy $[1,2]$ made the coupling of Adomian's polynomials with the correction functional (VIMAP) of the VIM and applied this reliable version for solving Riccati differential and Klein Gordon equations. In a later work, Noor and Mohyud-Din [62, 64, 74] exploited this concept for solving various singular and nonsingular boundary and initial value problems. Recently, Ghorbani et al. [13, 14] introduced He's polynomials (which are calculated from He's homotopy perturbation method) by splitting the nonlinear term and also proved that He's polynomials are fully compatible with Adomian's polynomials but are easier to calculate and are more user friendly. More recently, Noor and Mohyud-Din [60, 6669, 72, 73] combined He's polynomials and correction functional of the VIM and applied this reliable version (VIMHP) to a number of physical problems. It has been observed $[60,66-69,72,73]$ that the modification based on He's polynomials (VIMHP) which was developed by Noor and Mohyud-Din is much easier to implement as compared to the one (VIMAP) where the so-called Adomian's polynomials along with their complexities are used. The basic motivation of the present study is the review of these very powerful and reliable techniques which have been originated by He for solving various nonlinear initial and boundary value problems of diversified physical nature. Several examples are given to reveal the efficiency and potential of these relatively new techniques. We have also pointed out that the techniques discussed in this paper can be extended for solving obstacle, free, moving, and contact problems, which arise in various fields of pure and engineering sciences. This is another aspect of future research work. The interested readers are advised to explore this avenue for innovative and novel applications of these techniques. 


\section{Exp-Function Method}

Consider the general nonlinear partial differential equation of the type

$$
P\left(u, u_{t}, u_{x}, u_{t t}, u_{x x}, u_{x x x x}, \ldots\right)=0 .
$$

Using a transformation

$$
\eta=k x+\omega t
$$

where $k$ and $\omega$ are constants, we can rewrite (2.1) in the following nonlinear ODE;

$$
Q\left(u, u^{\prime}, u^{\prime \prime}, u^{\prime \prime \prime}, u^{(\mathrm{iv})}, \ldots\right)=0
$$

according to the exp-function method, which was developed by $\mathrm{He}$ and $\mathrm{Wu}$ [39], we assume that the wave solutions can be expressed in the following form

$$
u(\eta)=\frac{\sum_{n=-c}^{d} a_{n} \exp [n \eta]}{\sum_{m=-p}^{q} b_{m} \exp [m \eta]}
$$

where $p, q, c$, and $d$ are positive integers which are known to be further determined, $a_{n}$ and $b_{m}$ are unknown constants. We can rewrite (2.4) in the following equivalent form:

$$
u(\eta)=\frac{a_{c} \exp [c \eta]+\cdots+a_{-d} \exp [-d \eta]}{b_{p} \exp [p \eta]+\cdots+b_{-q} \exp [-q \eta]}
$$

This equivalent formulation plays an important and fundamental part for finding the analytic solution of problems $[5,17,39,40,53,54,58,59,75,76,84-87,97-100]$. To determine the value of $c$ and $p$, we balance the linear term of highest order of (2.4) with the highest order nonlinear term. Similarly, to determine the value of $d$ and $q$, we balance the linear term of lowest order of (2.3) with lowest order nonlinear term.

Example 2.1 (see [58]). Consider the ZK-MEW (2.6)

$$
u_{t}+a(u)^{3}{ }_{x}+\left(b u_{x t}+r u_{y y}\right)_{x}=0
$$

Introducing a transformation as $\eta=k x+\omega y+\rho t$, we can covert (2.6) into ordinary differential equations

$$
\rho u^{\prime}+3 a k u^{2} u^{\prime}+\left(b k^{2} \rho+r k \omega^{2}\right) u^{\prime \prime \prime}=0,
$$


where the prime denotes the derivative with respect to $\eta$. The trial solution of the (2.7) can be expressed as follows, as shown in (2.6):

$$
u(\eta)=\frac{a_{c} \exp [c \eta]+\cdots+a_{-d} \exp [-d \eta]}{b_{p} \exp [p \eta]+\cdots+b_{-q} \exp [-q \eta]}
$$

To determine the value of $c$ and $p$, we balance the linear term of highest order of (2.7) with the highest order nonlinear term, we obtain

$$
p=c, \quad d=q .
$$

Case 1. We can freely choose the values of $p, c, d$ but we will illustrate that the final solution does not strongly depend upon the choice of values of $c$ and $d$. For simplicity, we set $p=c=1$ and $q=d=1$, then the trial solution yields

$$
u(\eta)=\frac{a_{1} \exp [\eta]+a_{0}+a_{-1} \exp [-\eta]}{b_{1} \exp [\eta]+a_{0}+b_{-1} \exp [-\eta]}
$$

Substituting (2.10) into (2.7), we have

$$
\frac{1}{A}\left[c_{3} \exp (3 \eta)+c_{2} \exp (2 \eta)+c_{1} \exp (\eta)+c_{0}+c_{-1} \exp (-\eta)+c_{-2} \exp (-2 \eta)+c_{-3} \exp (-3 \eta)\right]=0
$$

where $A=\left(b_{1} \exp (\eta)+b_{0}+b_{-1} \exp (-\eta)\right)^{4}, c_{i}(i=-3, \ldots, 0, \ldots, 3)$ are constants obtained by Maple 11. Equating the coefficients of $\exp (n \eta)$ to be zero, we obtain

$$
\left\{c_{-3}=0, c_{-2}=0, c_{-1}=0, c_{0}=0, c_{1}=0, c_{2}=0, c_{3}=0\right\} .
$$

Solution of (2.12) will yield

$$
b_{1}=\frac{1}{8} \frac{a_{0}^{2} a\left(b k^{2}+1\right)}{b_{-1} r \omega}, \quad b_{-1}=b_{-1}, \quad a_{0}=a_{0}, \quad \rho=-\frac{k r \omega^{2}}{\left(b k^{2}+1\right)}, \quad a_{-1}=0, \quad a_{1}=0, \quad b_{0}=0
$$

We, therefore, obtained the following generalized solitary solution $u(x, y, t)$ of $(2.6)$ as follows:

$$
u(x, y, t)=\frac{a_{0}}{1 / 8\left(a_{0}^{2} a\left(1+b k^{2}\right)\right) /\left(b_{-1} r \omega^{2}\right) e^{(k x+\omega y+\rho t)}+b_{-1} e^{-(k x+\omega y+\rho t)}}
$$

where $\rho=-k r \omega^{2} /\left(b k^{2}+1\right), a_{0}, b_{-1}, a, b, k, r$, and $\omega$ are real numbers. 


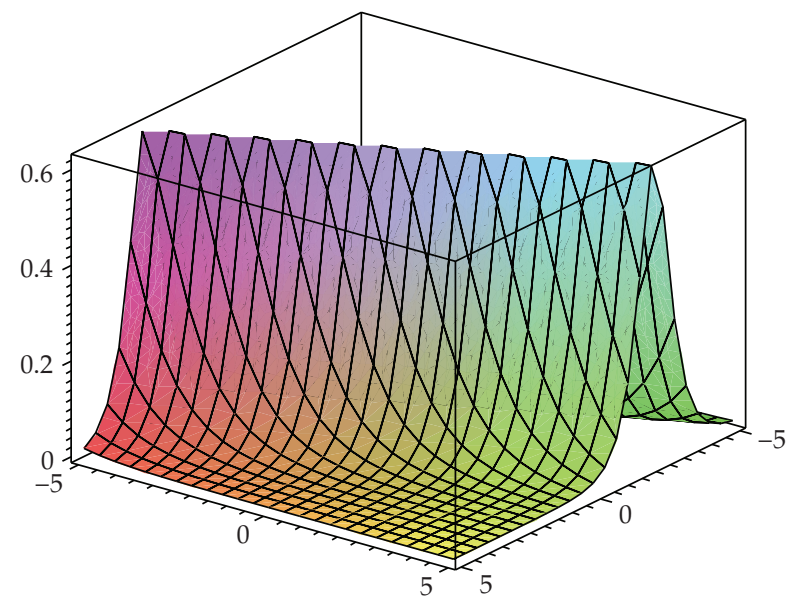

Figure 1

Figure 1 depicts the soliton solutions of (2.6), when $a_{0}=b_{-1}=a=b=r=k=1$. In case $k$ and $\omega$ are imaginary numbers, the obtained soliton solutions can be converted into periodic solutions or compact-like solutions. Therefore, we write $k=i K$ and $\omega=i W$ consequently, (2.14) becomes

$$
u(x, y, t)=\frac{a_{0}}{-1 / 8\left(a_{0}^{2} a\left(1-b K^{2}\right)\right) /\left(b_{-1} r W^{2}\right) e^{(i K x+i W y+\rho t)}+b_{-1} e^{-(i K x+i W y+\rho t)}} .
$$

The above expression can be rewritten in expanded form:

$$
u(x, y, t)=\frac{-8 a_{0} b_{-1} r W^{2}\left[\begin{array}{c}
\cos \left(\frac{\mathcal{A}}{b K^{2}-1}\right)\left[-a b a_{0}{ }^{2} K^{2}+a a_{0}{ }^{2}-8 r W^{2} b_{-1}{ }^{2}\right] \\
+i \sin \left(\frac{\mathcal{A}}{b K^{2}-1}\right)\left[-a b a_{0}{ }^{2} K^{2}+a a_{0}{ }^{2}+8 b_{-1}{ }^{2} r W^{2}\right]
\end{array}\right]}{\left[\begin{array}{c}
32 a b a_{0}{ }^{2} r b_{-1} W^{2} \cos (\mathcal{A})^{2}\left[K^{2}-1\right]+a^{2} b^{2} a_{0}{ }^{4} K^{4} \\
-2 a^{2} b a_{0}{ }^{4} K^{2}-16 a b a_{0}{ }^{2} b_{-1}{ }^{2} r K^{2} W^{2}+a^{2} a_{0}{ }^{4}+16 a a_{0}{ }^{2} b_{-1}{ }^{2} r W^{2}+64 b_{-1}{ }^{4} r^{2} W^{4}
\end{array}\right]},
$$

where $\mathcal{A}=-K^{3} b x+K x-W b K^{2} y+W y+K r W^{2} t$. If we search for periodic solutions or compact-like solutions, the imaginary part in (2.16) must be zero, hence

$$
u(x, y, t)=\frac{-8 a_{0} b_{-1} r W^{2}\left[\cos (\mathcal{A})\left[-a b a_{0}{ }^{2} K^{2}+a a_{0}{ }^{2}-8 r W^{2} b_{-1}{ }^{2}\right]\right]}{\left[\begin{array}{c}
32 a b a_{0}{ }^{2} r b_{-1} W^{2} \cos (\mathcal{A})^{2}\left[K^{2}-1\right]+a^{2} b^{2} a_{0}{ }^{4} K^{4} \\
-2 a^{2} b a_{0}{ }^{4} K^{2}-16 a b a_{0}{ }^{2} b_{-1}{ }^{2} r K^{2} W^{2}+a^{2} a_{0}{ }^{4}+16 a a_{0}{ }^{2} b_{-1}{ }^{2} r W^{2}+64 b_{-1}{ }^{4} r^{2} W^{4}
\end{array}\right]},
$$

Figure 2 depicts the periodic solutions of (2.6) when $a=b=a_{0}=b_{-1}=K=W=r=t=1$. 


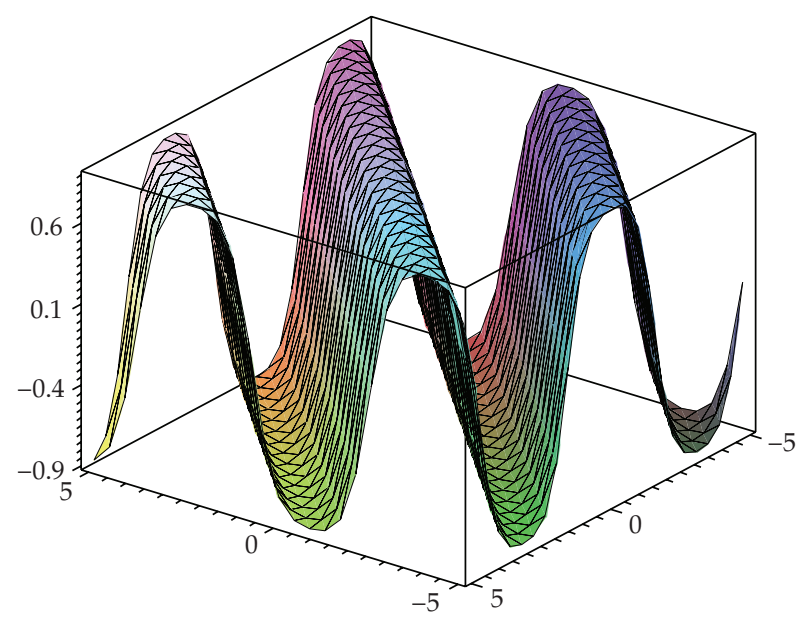

Figure 2

Case 2. If $p=c=2$, and $q=d=1$, then the trial solution, (2.6) reduces to

$$
u(\eta)=\frac{a_{2} \exp [2 \eta]+a_{1} \exp [\eta]+a_{0}+a_{-1} \exp [-\eta]}{b_{2} \exp [2 \eta]+b_{1} \exp [\eta]+b_{0}+b_{-1} \exp [-\eta]}
$$

Proceeding as before, we obtain

$$
\begin{gathered}
b_{2}=b_{2}, \quad b_{1}=0, \quad b_{0}=0, \quad a_{1}=0, \quad \omega=\omega, \quad b_{-1}=b_{-1}, \quad \rho=\frac{9 k r \omega^{2}}{\left(9 b k^{2}-2\right)} \\
a_{0}=0, \quad a_{2}=a_{2}, \quad b_{0}=0, \quad a_{-1}=-\frac{b_{-1} a_{2}}{b_{2}}, \quad \alpha=\frac{9 b_{2}^{2} r \omega^{2}}{\left(9 b k^{2}-2\right) a_{2}{ }^{2}} .
\end{gathered}
$$

Hence, we get the generalized solitary solutions $u(x, y, t)$ of (2.6) as follows:

$$
u(x, y, t)=-1+\frac{2 b_{-1}}{b_{1} e^{2\left(k x+\omega y-\left(9 k r \omega^{2} t\right) /\left(9 b k^{2}-2\right)\right)}+b_{-1}}
$$

where $b_{-1}, b_{1}, \omega$, and $\mathrm{k}$ are real numbers.

Remark 2.2. It is worth mentioning that the transformation $k=i k$ which is used to transform the solitary solutions to periodic or compacton-like solutions was first proposed by $\mathrm{He}$ and $\mathrm{Wu}[39]$ and is called the He-Wu transformation. Moreover, the interpretation of this transformation is given by He [17]. 


\section{Variational Iteration Method (VIM) and its Modifications}

To illustrate the basic concept of the He's VIM, we consider the following general differential equation:

$$
L u+N u=g(x)
$$

where $L$ is a linear operator, $N$ a nonlinear operator and $g(x)$ is the inhomogeneous term. According to variational iteration method $[1-7,11,17-24,44,49,50,60-64,66-74,77-80,88$, 90], we can construct a correction functional as follows:

$$
u_{n+1}(x)=u_{n}(x)+\int_{0}^{x} \lambda\left(L u_{n}(s)+N \tilde{u}_{n}(s)-g(s)\right) d s,
$$

where $\lambda$ is a Lagrange multiplier [17-24], which can be identified optimally via variational iteration method. The subscripts $n$ denote the $n$th approximation, $\tilde{u}_{n}$ is considered as a restricted variation. That is, $\delta \tilde{u}_{n}=0 ;(3.2)$ is called a correction functional. The solution of the linear problems can be solved in a single iteration step due to the exact identification of the Lagrange multiplier. The principles of variational iteration method and its applicability for various kinds of differential equations are given in [17-24]. In this method, it is required first to determine the Lagrange multiplier $\lambda$ optimally. The successive approximation $u_{n+1}, n \geq 0$ of the solution $u$ will be readily obtained upon using the determined Lagrange multiplier and any selective function $u_{0}$, consequently, the solution is given by $u=\lim _{n \rightarrow \infty} u_{n}$. We summarize some useful iteration formulae $[23,24]$ which would be used in the subsequent section:

$$
\begin{gathered}
u^{\prime}+f\left(u, u^{\prime}\right)=0, \\
u_{n+1}(t)=u_{n}(t)-\int_{0}^{t}\left(u_{n}^{I}(s)+f\left(u_{n}, u_{n}^{I}\right)\right) d s . \\
u^{\prime \prime}+f\left(u, u^{\prime}, u^{\prime \prime}\right)=0, \\
u_{n+1}(t)=u_{n}(t)+\int_{0}^{t}(s-t)\left(u_{n}^{I I}(s)+f\left(u_{n}, u_{n}^{I}, u_{n}^{I I}\right)\right) d s . \\
u^{\prime \prime \prime}+f\left(u, u^{\prime}, u^{\prime \prime}, u^{\prime \prime \prime}\right)=0, \\
u_{n+1}(t)=u_{n}(t)-\int_{0}^{t} \frac{1}{2 !}(s-t)^{2}\left(u_{n}^{I I I}(s)+f\left(u_{n}, u_{n}^{I}, u_{n}^{I I}, u_{n}^{I I I}\right)\right) d s . \\
u^{(i v)}+f\left(u, u^{\prime}, u^{\prime \prime}, u^{\prime \prime \prime}, u^{(i v)}\right)=0, \\
u_{n+1}(t)=u_{n}(t)+\int_{0}^{t} \frac{1}{3 !}(s-t)^{3}\left(u_{n}^{(i v)}(s)+f\left(u_{n}, u_{n}^{I}, u_{n}^{I I}, u_{n}^{I I I}, u_{n}^{(i v)}\right)\right) d s .
\end{gathered}
$$




$$
\begin{gathered}
u^{(n)}+f\left(u, u^{\prime}, u^{\prime \prime}, u^{\prime \prime \prime}, u^{(i v)}, \ldots, u^{(n)}\right)=0 \\
u_{n+1}(t)=u_{n}(t)+(-1)^{n} \int_{0}^{t} \frac{1}{(n-1) !}(s-t)^{n-1}\left(u_{n}^{(n)}(s)+\left(u_{n}, u_{n}^{I}, u_{n}^{I I}, u_{n}^{I I I}, u_{n}^{(i v)}, \ldots, u_{n}^{(n)}\right)\right) d s .
\end{gathered}
$$

\subsection{Variational Iteration Method Using He's Polynomials (VIMHP)}

This modified version of variational iteration method $[60,66-69,72,73]$ is obtained by the elegant coupling of correction functional (2.7) of variational iteration method (VIM) with He's polynomials and is given by

$$
\sum_{n=0}^{\infty} p^{(n)} u_{n}=u_{0}(x)+p \int_{0}^{x} \lambda(s)\left(\sum_{n=0}^{\infty} p^{(n)} L\left(u_{n}\right)+\sum_{n=0}^{\infty} p^{(n)} N\left(\tilde{u}_{n}\right)\right) d s-\int_{0}^{x} \lambda(s) g(s) d s
$$

comparisons of like powers of $p$ give solutions of various orders.

\subsection{Variational Iteration Method Using Adomian's Polynomials (VIMAP)}

This modified version of VIM is obtained by the coupling of correction functional (2.3) of VIM with Adomian's polynomials $[1,2,62,64,70,74]$ and is given by

$$
u_{n+1}(x)=u_{n}(x)+\int_{0}^{t} \lambda\left(L u_{n}(x)+\sum_{n=0}^{\infty} A_{n}-g(x)\right) d x
$$

where $A_{n}$ are the so-called Adomian's polynomials and are calculated for various classes of nonlinearities by using the specific algorithm developed in [81-83].

Example 3.1 (see [61]). Consider the following singularly perturbed sixth-order Boussinesq equation

$$
u_{t t}=u_{x x}+\left(u^{2}\right)_{x x}-u_{x x x x}+\frac{1}{2} u_{x x x x x x}
$$

with initial conditions

$$
u(x, 0)=-\frac{105}{169} \operatorname{sech}^{4}\left(\frac{x}{\sqrt{26}}\right), \quad u_{t}(x, 0)=\frac{-210 \sqrt{194 / 13} \operatorname{sech}^{4}(x / \sqrt{26}) \tanh (x / \sqrt{26})}{2197} .
$$


The exact solution of the problem is given as

$$
u(x, t)=-\frac{105}{169} \operatorname{sech}^{4}\left[\sqrt{\frac{1}{26}}\left(x-\sqrt{\frac{97}{169}} t\right)\right]
$$

The correction functional is given by

$$
\begin{aligned}
u_{n+1}(x, t)=- & \frac{105}{169} \operatorname{sech}^{4}\left(\frac{x}{\sqrt{26}}\right)+\frac{-210 \sqrt{194 / 13} \operatorname{sech}^{4}(x / \sqrt{26}) \tanh (x / \sqrt{26})}{2197} t \\
& +\int_{0}^{t} \lambda(s)\left(\frac{\partial^{2} u_{n}}{\partial t^{2}}-\left(\left(\widetilde{u}_{n}\right)_{x x}+\left(\tilde{u}_{n}^{2}\right)_{x x}-\left(\tilde{u}_{n}\right)_{x x x x}+\frac{1}{2}\left(\widetilde{u}_{n}\right)_{x x x x x x}\right)\right) d s .
\end{aligned}
$$

Making the correction functional stationary, the Lagrange multiplier can easily be identified as $\lambda(s)=(s-x)$, we get the following iterative formula

$$
\begin{aligned}
u_{n+1}(x, t)=- & \frac{105}{169} \operatorname{sech}^{4}\left(\frac{x}{\sqrt{26}}\right)+\frac{-210 \sqrt{194 / 13} \operatorname{sech}^{4}(x / \sqrt{26}) \tanh (x / \sqrt{26})}{2197} t \\
& +\int_{0}^{t}(s-x)\left(\frac{\partial^{2} u_{n}}{\partial t^{2}}-\left(\left(u_{n}\right)_{x x}+\left(u_{n}{ }^{2}\right)_{x x}-\left(u_{n}\right)_{x x x x}+\frac{1}{2}\left(u_{n}\right)_{x x x x x x}\right)\right) d s .
\end{aligned}
$$

Consequently, following approximants are obtained

$$
\begin{aligned}
u_{0}(x, t)= & -\frac{105}{169} \operatorname{sech}^{4}\left(\frac{x}{\sqrt{26}}\right) \\
u_{1}(x, t)= & -\frac{105}{169} \operatorname{sech}^{4}\left(\frac{x}{\sqrt{26}}\right)-\frac{105 \sqrt{194 / 13} \operatorname{sech}^{6}(x / \sqrt{26}) \sinh (\sqrt{2} x / \sqrt{13})}{2197} t \\
& -\frac{105}{371293}\left(-291+194 \cosh \left(\frac{\sqrt{2} x}{\sqrt{13}}\right)\right) \operatorname{sech}^{6} \frac{x}{\sqrt{26}} t^{2}
\end{aligned}
$$


Table 1: Error estimates.

\begin{tabular}{|c|c|c|c|c|c|c|}
\hline$x_{i}$ & $\begin{array}{c}t_{j} \\
0.01\end{array}$ & 0.02 & 0.04 & 0.1 & 0.2 & 0.5 \\
\hline-1 & 7.77156 E-16 & 1.36557 E-14 & 8.57869 E-13 & $2.09264 \mathrm{E}-10$ & $1.33823 \mathrm{E}-8$ & 3.25944 E-6 \\
\hline-0.8 & 1.11022 E-16 & 1.99840 E-15 & $1.12688 \mathrm{E}-13$ & $2.73880 \mathrm{E}-11$ & 1.74288 E-9 & 4.14094 E-7 \\
\hline-0.6 & 2.22045 E-16 & 1.09912 E-14 & 7.28861 E-13 & $1.78030 \mathrm{E}-10$ & 1.14025 E-8 & 2.79028 E-6 \\
\hline-0.4 & 1.11022 E-16 & 2.32037 E-14 & 1.50302 E-12 & 3.67002 E-10 & 2.34944 E-8 & 5.74091 E-6 \\
\hline-0.2 & 6.66134 E-16 & 3.23075 E-14 & 2.04747 E-12 & 4.99918 E-10 & 3.19983 E-9 & 7.81509 E-6 \\
\hline 0 & 4.44089 E-16 & 3.49720 E-14 & 2.24365 E-12 & 5.47741 E-10 & 3.50559 E-8 & 8.55935 E-6 \\
\hline 0.2 & 5.55112 E-16 & 3.19744 E-14 & 2.04714 E-12 & 4.99820 E-10 & 3.19858 E-8 & 7.80749 E-6 \\
\hline 0.4 & 3.33067 E-16 & 2.32037 E-14 & 1.50324 E-12 & 3.66815 E-10 & 2.34706 E-8 & 5.72641 E-6 \\
\hline 0.6 & 3.33067 E-16 & 1.12133 E-14 & 7.28528 E-12 & 1.77772 E-10 & 1.13695 E-8 & 2.77022 E-6 \\
\hline 0.8 & 3.33067 E-16 & 1.99840 E-15 & $1.13132 \mathrm{E}-13$ & 2.76944 E-11 & 1.78208 E-9 & 4.41936 E-7 \\
\hline 1 & 7.77156 E-16 & 1.38778 E-14 & 8.58313 E-13 & $2.09593 \mathrm{E}-10$ & 1.34244 E-8 & 3.28504 E-6 \\
\hline
\end{tabular}

The series solution is given by

$$
\begin{aligned}
u(x, t)=- & \frac{105}{169} \operatorname{sech}^{4}\left(\frac{x}{\sqrt{26}}\right)-\frac{105 \sqrt{194 / 13} \operatorname{sech}^{6}(x / \sqrt{26}) \sinh (\sqrt{2} x / \sqrt{13})}{2197} t \\
& -\frac{105}{371293}\left(-291+194 \cosh \left(\frac{\sqrt{2} x}{\sqrt{13}}\right)\right) \operatorname{sech}^{6} \frac{x}{\sqrt{26}} t^{2} \\
& +\frac{395 \operatorname{sech}^{7} \frac{x}{\sqrt{26}}\left(10816 \sqrt{2522} \sinh \frac{x}{\sqrt{26}}-1664 \sqrt{2522} \sinh \frac{3 x}{\sqrt{26}}\right) t^{3}}{} \\
& +\left(-334200 \operatorname{sech}^{5}\left(\frac{x}{\sqrt{26}}\right)+354247 \cosh \left(\frac{2}{\sqrt{13}} x\right) \operatorname{sech}^{5}\left(\frac{x}{\sqrt{26}}\right)\right. \\
& \left.-47164 \cosh ^{2}\left(\frac{2 \sqrt{2}}{\sqrt{13}} x\right) \operatorname{sech}^{5}\left(\frac{x}{\sqrt{26}}\right)\right) t^{4} \\
& +\left(3201 \cosh ^{3}\left(\frac{3 \sqrt{2}}{\sqrt{13}} x\right) \operatorname{sech}^{5}\left(\frac{x}{\sqrt{26}}\right)-388 \cosh \left(\frac{4 \sqrt{2}}{\sqrt{13}} x\right) \operatorname{sech}^{5}\left(\frac{x}{\sqrt{26}}\right)\right) t^{4} \\
& +\cdots,
\end{aligned}
$$

Table 1 exhibits the absolute error between the exact and the series solutions. Higher accuracy can be obtained by introducing some more components of the series solution.

Example 3.2 (see [72]). Consider the following Helmholtz equation:

$$
\frac{\partial^{2} u}{\partial x^{2}}+\frac{\partial^{2} u(x, y)}{\partial y^{2}}-u(x, y)=0
$$




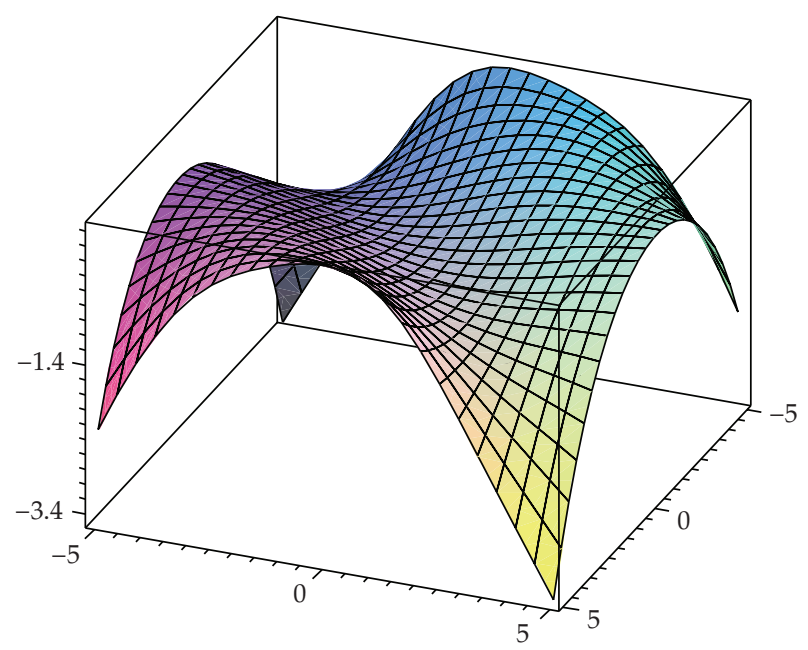

Figure 3

with initial conditions

$$
u(0, y)=y, \quad u_{x}(0, y)=y+\cosh y .
$$

The correction functional is given as

$$
y_{n+1}(x)=y_{n}(x)+\int_{0}^{x} \lambda(s)\left(\frac{\partial^{2} u_{n}}{\partial x^{2}}+\frac{\partial^{2} \tilde{u}_{n}(x, y)}{\partial y^{2}}-\tilde{u}_{n}(x, y)\right) d s .
$$

Making the correction functional stationary, the Lagrange multiplier can be identified as $\lambda(s)=(s-x)$, we obtained

$$
y_{n+1}(x)=y_{n}(x)+\int_{0}^{x}(s-x)\left(\frac{\partial^{2} u_{n}}{\partial x^{2}}+\frac{\partial^{2} u_{n}(x, y)}{\partial y^{2}}-u_{n}(x, y)\right) d s
$$

Applying the variational iteration method using He's polynomials (VIMHP), we get

$$
\begin{gathered}
y_{0}+p y_{1}+p^{2} y_{2}+\cdots=y_{n}(x)+p \int_{0}^{x}(s-x)\left(\frac{\partial^{2} u_{0}}{\partial x^{2}}+p \frac{\partial^{2} u_{1}}{\partial x^{2}}+p^{2} \frac{\partial^{2} u_{2}}{\partial x^{2}}+\cdots\right) d s \\
+p \int_{0}^{x}(s-x)( \\
\left(\frac{\partial^{2} u_{0}}{\partial y^{2}}+p \frac{\partial^{2} u_{1}}{\partial y^{2}}+p^{2} \frac{\partial^{2} u_{2}}{\partial y^{2}}+\cdots\right) \\
\left.-\left(u_{0}+p u_{1}+p^{2} u_{2}+\cdots\right)\right) d s .
\end{gathered}
$$


Table 2: Error estimates.

\begin{tabular}{lcccccc}
\hline $\mathrm{X}$ & $\mathrm{Y}$ & Exact solution & HPM & ADM & VIMHP & Absolute error \\
\hline-1 & -1 & -1.9109600760 & -1.9097472990 & -1.9097472990 & -1.9097472990 & 0.0012127770 \\
-0.8 & -0.8 & -1.4294111280 & -1.491500900 & -1.491500900 & -1.491500900 & 0.0002610380 \\
-0.6 & -0.6 & -1.0405661130 & -1.0405303310 & -1.0405303310 & -1.0405303310 & 0.0000357820 \\
-0.4 & -0.4 & -.7005569670 & -0.7005548150 & -0.7005548150 & -0.7005548150 & 0.0000021520 \\
-0.2 & -0.2 & -.367755010 & -0.3677594840 & -0.3677594840 & -0.3677594840 & 0.0000000170 \\
0.0 & -0.0 & 0.0000000000 & 0.0000000000 & 0.0000000000 & 0.0000000000 & 0.0000000000 \\
0.2 & 0.2 & 0.4482939020 & 0.4482938840 & 0.4482938840 & 0.4482938840 & 0.0000000180 \\
0.4 & 0.4 & 1.0291588280 & 1.0291564150 & 1.0291564150 & 1.0291564150 & 0.0000024130 \\
0.6 & 0.6 & 1.8045504110 & 1.8045079310 & 1.8045079310 & 1.8045079310 & 0.0000424800 \\
0.8 & 0.8 & 2.850380700 & 2.8500524900 & 2.8500524900 & 2.8500524900 & 0.0003282100 \\
1.0 & 1.0 & 4.2613624630 & 4.2597472990 & 4.2597472990 & 4.2597472990 & 0.0016151640 \\
\hline
\end{tabular}

Comparing the coefficient of like powers of $p$, following approximants are obtained:

$$
\begin{aligned}
& p^{(0)}: u_{0}(x, y)=y(1+x)+x \cosh y, \\
& p^{(1)}: u_{1}(x, y)=y(1+x)+x \cosh y+\frac{1}{2 !} x^{2} y+\frac{1}{3 !} x^{3} y \\
& p^{(2)}: u_{2}(x, y)=y(1+x)+x \cosh y+\frac{1}{2 !} x^{2} y+\frac{1}{3 !} x^{3} y+\frac{1}{4 !} x^{4} y+\frac{1}{5 !} x^{5} y,
\end{aligned}
$$

The solution is given as

$$
u(x, y)=y \exp (x)+x \cosh (y)
$$

Table 2 exhibits the approximate solution obtained by using the HPM, ADM, and VIMHP. It is clear that the obtained results are in high agreement with the exact solutions. Higher accuracy can be obtained by using more terms.

Example 3.3 (see [62]). Consider the following nonlinear Schrödinger equation

$$
i u_{t}+u_{x x}-2 u|u|^{2}=0
$$

with initial conditions

$$
u(x, 0)=e^{i x}
$$

The correction functional is given as

$$
u_{n+1}(x, t)=e^{i x}+\int_{0}^{t} \lambda(s)\left(\frac{\partial u_{n}}{\partial s}-i\left(\left(\tilde{u}_{n}\right)_{x x}-2 \tilde{u}_{n}\left|\tilde{u}_{n}\right|^{2}\right)\right) d s .
$$




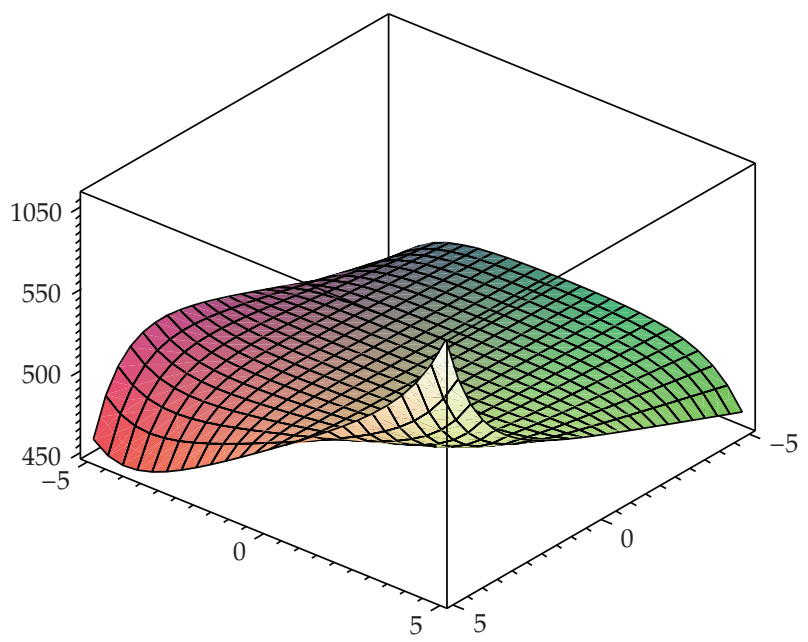

Figure 4: Depicts series solutions $u(x, y)$.

Making the correction functional stationary, the Lagrange multipliers can be identified as $\lambda(s)=-1$, consequently

$$
u_{n+1}(x, t)=e^{i x}-\int_{0}^{t}\left(\frac{\partial u_{n}}{\partial s}-i\left(\left(u_{n}\right)_{x x}-2 u_{n}\left|u_{n}\right|^{2}\right)\right) d s
$$

Applying the variational iteration method using Adomian's polynomials (VIMAP):

$$
u_{n+1}(x, t)=e^{i x}-\int_{0}^{t}\left(\frac{\partial u_{n}}{\partial s}-i\left(\left(u_{n}\right)_{x x}-2 \sum_{n=0}^{\infty} A_{n}\right)\right) d s,
$$

where $A_{n}$ are the so-called Adomian's polynomials. First few Adomian's polynomials for nonlinear Schrödinger equation are as follows:

$$
\begin{gathered}
A_{0}=u_{o}^{2} \bar{u}_{0}, \\
A_{1}=2 u_{0} u_{1} \bar{u}_{0}+u_{o}^{2} \bar{u}_{1}, \\
A_{2}=2 u_{0} u_{2} \bar{u}_{0}+u_{1}^{2} \bar{u}_{0}+2 u_{0} u_{1} \bar{u}_{2}+u_{o}^{2} \bar{u}_{2,} \\
A_{3}=2 u_{0} u_{3} \bar{u}_{0}+u_{1}^{2} \bar{u}_{1}+2 u_{1} u_{2} \bar{u}_{0}+u_{o}^{2} \bar{u}_{3}+2 u_{0} u_{2} \bar{u}_{1}+2 u_{0} u_{1} \bar{u}_{2},
\end{gathered}
$$


Employing these polynomials in the above iterative scheme, following approximants are obtained:

$$
\begin{gathered}
u_{0}(x, t)=e^{i x}, \\
u_{1}(x, t)=e^{i x}(1-3 i t), \\
u_{2}(x, t)=e^{i x}\left(1-3 i t-\frac{9}{2 !} t^{2}\right), \psi \\
u_{3}(x, t)=e^{i x}\left(1-3 i t-\frac{9}{2 !} t^{2}+\frac{9}{2 !} i t^{3}\right), \psi
\end{gathered}
$$

The solution in a series form is given by

$$
u(x, t)=e^{i x}\left(1-3 i t+\frac{(3 i t)^{2}}{2 !} t^{2}-\frac{(3 i t)^{3}}{3 !} t^{3}+\frac{(3 i t)^{4}}{4 !} t^{4}-\cdots\right)
$$

and in a closed form by

$$
u(x, t)=e^{i(x-3 t)} .
$$

Remark 3.4. It is worth mentioningthat although both the modified versions of variational iteration method (VIM) are compatible yet the modification based upon He's polynomials (VIMHP) is much easier to implement and is more user friendly as compared to VIMAP where Adomian's polynomials along with their complexities are used.

\section{Homotopy Perturbation Method (HPM) and He's Polynomials}

To explain the He's homotopy perturbation method, we consider a general equation of the type,

$$
L(u)=0,
$$

where $L$ is any integral or differential operator. We define a convex homotopy $H(u, p)$ by

$$
H(u, p)=(1-p) F(u)+p L(u)
$$

where $F(u)$ is a functional operator with known solutions $v_{0}$, which can be obtained easily. It is clear that, for

$$
H(u, p)=0,
$$


we have

$$
H(u, 0)=F(u), \quad H(u, 1)=L(u) .
$$

This shows that $H(u, p)$ continuously traces an implicitly defined curve from a starting point $H\left(v_{0}, 0\right)$ to a solution function $H(f, 1)$. The embedding parameter monotonically increases from zero to unit as the trivial problem $F(u)=0$ continuously deforms the original problem $L(u)=0$. The embedding parameter $p \in(0,1]$ can be considered as an expanding parameter $[13,14,17,18,26-38,51,52,55-57,60,65-69,72,73,89,91-93,96]$. The homotopy perturbation method uses the homotopy parameter $p$ as an expanding parameter $[17,18,26-38]$ to obtain

$$
u=\sum_{i=0}^{\infty} p^{i} u_{i}=u_{0}+p u_{1}+p^{2} u_{2}+p^{3} u_{3}+\cdots
$$

if $p \rightarrow 1$, then (4.5) corresponds to (4.2) and becomes the approximate solution of the form,

$$
f=\lim _{p \rightarrow 1} u=\sum_{i=0}^{\infty} u_{i}
$$

It is well known that series (4.5) is convergent for most of the cases and also the rate of convergence is dependent on $\mathrm{L}(\mathrm{u})$; see $[17,18,26-38]$. We assume that (4.6) has a unique solution. The comparisons of like powers of $p$ give solutions of various orders. In sum, according to $[13,14]$, He's HPM considers the nonlinear term $N(u)$ as

$$
N(u)=\sum_{i=0}^{\infty} p^{i} H_{i}=H_{0}+p H_{1}+p^{2} H_{2}+\cdots,
$$

where $H_{n}$ 's are the so-called He's polynomials $[13,14]$, which can be calculated by using the formula

$$
H_{n}\left(u_{0}, \ldots, u_{n}\right)=\frac{1}{n !} \frac{\partial^{n}}{\partial p^{n}}\left(N\left(\sum_{i=0}^{n} p^{i} u_{i}\right)\right)_{p=0}, \quad n=0,1,2, \ldots
$$

Example 4.1 (see [51]). Consider the following seventh-order generalized KdV (SOG-KdV) equation

$$
u_{t}+u u_{x}+u_{x x x}-u_{x x x x x}+\sigma u_{x x x x x x x}=0,
$$

where $\sigma=\beta \delta / \gamma^{2}$, with initial conditions

$$
u(x, 0)=a_{0}+a_{6} \operatorname{sech}^{6}(k x),
$$


where $K=5 / \sqrt{1538}, a_{0}=c-18000 / 769^{2}, a_{6}=519750 / 769^{2}$, and $c$ is an arbitrary parameter. Applying the convex homotopy, we get

$$
\begin{gathered}
u_{0}+p u_{1}+p^{2} u_{2}+\cdots=u_{0}-p \int_{0}^{t}\left(\left(u_{0}+p u_{1}+p^{2} u_{2}+\cdots\right)\left(\frac{\partial u_{0}}{\partial x}+p \frac{\partial u_{1}}{\partial x}+p^{2} \frac{\partial u_{2}}{\partial x}+\cdots\right)\right) d s \\
-p \int_{0}^{t}\left(\left(\frac{\partial^{3} u_{0}}{\partial x^{3}}+p \frac{\partial^{3} u_{1}}{\partial x^{3}}+\cdots\right)-\left(\frac{\partial^{5} u_{0}}{\partial x^{5}}+p \frac{\partial^{5} u_{1}}{\partial x^{5}}+\cdots\right)\right. \\
\left.+\sigma\left(\frac{\partial^{7} u_{0}}{\partial x^{7}}+p \frac{\partial^{7} u_{1}}{\partial x^{7}}+\cdots\right)\right) d s
\end{gathered}
$$

Comparing the coefficient of like powers of $p$

$$
\begin{aligned}
p^{(0)}: u_{0}(x, t)=a_{0}+a_{6} & \operatorname{sech}^{6}(k x), \\
p^{(1)}: u_{1}(x, t)=\frac{3 a_{6} k t}{16}\{1 & 10 a_{0}+32 a_{6}-312 k^{2}-26400 k^{4}-9866112 \delta k^{6} \\
& +\left(15 a_{0}-256 k^{2}-10480 k^{4}+9932224 \delta k^{6}\right) \cosh (2 k x) \\
& +\left(6 a_{0}-8 k^{2}+14624 k^{4}-1443968 \delta k^{6}\right) \cosh (4 k x) \\
& \left.+\left(a_{0}+36 k^{2}-1296 k^{4}+46656 \delta k^{6}\right) \cosh (6 k x)\right\} \operatorname{sech}^{12}(k x) \tanh (k x),
\end{aligned}
$$

where $p^{i} s$ are the He's polynomials. The series solution is given by

$$
\begin{aligned}
u(x, t)=a_{0} & +a_{6} \operatorname{sech}^{6}(k x)+\frac{3 a_{6} k t}{16}\left\{10 a_{0}+32 a_{6}-312 k^{2}-26400 k^{4}-9866112 \delta k^{6}\right. \\
& +\left(15 a_{0}-256 k^{2}-10480 k^{4}+9932224 \delta k^{6}\right) \cosh (2 k x) \\
& +\left(6 a_{0}-8 k^{2}+14624 k^{4}-1443968 \delta k^{6}\right) \cosh (4 k x) \\
& \left.+\left(a_{0}+36 k^{2}-1296 k^{4}+46656 \delta k^{6}\right) \cosh (6 k x)\right\} \operatorname{sech}^{12}(k x) \tanh (k x) \\
& -\frac{3 a_{6} k t^{2}}{4096}\left[1320 a_{0}^{2}+6400 a_{0} a_{6}+40960 a_{6}^{2}-93120 a_{0} k^{2}-642560 a_{6} k^{2}\right. \\
& +3592320 k^{4}-718464 a_{0} k^{4}-223897600 a_{6} k^{4}+1066859520 k^{6} \\
& -1066859520 a_{0} \delta k^{6}-371744399360 a_{6} \delta k^{6}+151760209920 k^{8} \\
& +303520419840 \delta k^{10}+186385174609920 \delta^{2} k^{12}
\end{aligned}
$$




$$
\begin{aligned}
& +\left(2079 a_{0}^{2}+6912 a_{0} a_{6}-36864 a_{6}^{2}-124488 a_{0} k^{2}-291840 a_{6} k^{2}\right. \\
& +3662064 k^{4}-7324128 a_{0} k^{4}+83202048 a_{6} k^{4}+643886208 k^{6}-643886208 a_{0} \delta k^{6} \\
& +520393113600 a_{6} \delta k^{6}+10077306624 k^{8}+20154613248 \delta k^{8}-115427212572672 \delta k^{10} \\
& \left.-318167001264623616 \delta^{2} k^{12}\right) \cosh (2 k x)+\left(924 a_{0}^{2}-1536 a_{0} a_{6}-14112 a_{0} k^{2}\right. \\
& +592896 a_{6} k^{2}-1544256 k^{4}+3088512 a_{0} k^{4}+208736256 a_{6} k^{4}-1003580928 k^{6} \\
& +1003580928 a_{0} \delta k^{6}-173675003904 a_{6} \delta k^{6}-180653147136 k^{8} \\
& -361306294272 \delta k^{8}-159045011693568 \delta k^{10} \\
& \left.+129148767835766784 \delta^{2} k^{12}\right) \cosh (4 k x)+\left(77 a_{0}^{2}-2816 a_{0} a_{6}+36904 a_{0} k^{2}\right. \\
& -2283568 k^{4}+4567136 a_{0} \delta k^{6}+4399738112 k^{8}+8799476224 \delta k^{8} \\
& \left.+114650957797376 \delta k^{10}-27618434663723008 \delta^{2} k^{12}\right) \cosh (6 k x)-\left(168 a_{0}^{2}\right. \\
& +768 a_{0} a_{6}-25536 a_{0} k^{2}+69120 a_{6} k^{2}+594048 k^{4}-1188096 a_{0} k^{4}-8460288 a_{6} k^{4} \\
& -118291504 k^{6}+118293504 a_{0} \delta k^{6}+1164533760 a_{6} \delta k^{6}-35208886272 k^{8} \\
& \left.+70417772544 \delta k^{8}+25860049453056 \delta k^{10}-2901989324193792 \delta^{2} k^{12}\right) \cosh (8 k x) \\
& \left.+61463535616 \delta k^{10}-2068416315392 \delta^{2} k^{12}\right) \cosh (12 k x)-\left(3 a_{0}^{2}+216 a_{0} k^{2}\right. \\
& +3888 k^{4}-7776 a_{0} k^{4}-279936 k^{6}+279936 a \delta k^{6}+5038848 k^{8}+10077696 \delta k^{8} \\
& + \\
& + \\
& + \\
& +
\end{aligned}
$$

The closed form solution is given as

$$
u(x, t)=a_{0}+a_{6} \operatorname{sech}^{6}(k(x-c t)),
$$

where $k=5 / \sqrt{1538}, a_{0}=c-18000 / 769^{2}, a_{6}=519750 / 769^{2}$, and $c$ is an arbitrary parameter. 
Table 3: Error Estimates $(\boldsymbol{\delta}=.01)$.

\begin{tabular}{lcccc}
\hline$x$ & & Error $t_{j}$ \\
$j=.05$ & $j=.1$ \\
\hline-1.0 & $j=.001$ & $j=.01$ & $5.6 \times 10^{-3}$ & $1.9 \times 10^{-2}$ \\
-.8 & $3.1 \times 10^{-5}$ & $4.6 \times 10^{-4}$ & $5.2 \times 10^{-3}$ & $1.8 \times 10^{-2}$ \\
-.6 & $2.6 \times 10^{-5}$ & $4.0 \times 10^{-4}$ & $4.7 \times 10^{-3}$ & $1.7 \times 10^{-2}$ \\
-.4 & $2.0 \times 10^{-5}$ & $3.4 \times 10^{-4}$ & $4.3 \times 10^{-3}$ & $1.5 \times 10^{-2}$ \\
-.2 & $1.4 \times 10^{-5}$ & $2.7 \times 10^{-4}$ & $3.9 \times 10^{-3}$ & $1.4 \times 10^{-2}$ \\
0.0 & $7.8 \times 10^{-6}$ & $2.0 \times 10^{-4}$ & $3.5 \times 10^{-3}$ & $1.4 \times 10^{-2}$ \\
.2 & $1.4 \times 10^{-6}$ & $1.4 \times 10^{-4}$ & $3.2 \times 10^{-3}$ & $1.3 \times 10^{-2}$ \\
.4 & $5.0 \times 10^{-6}$ & $7.8 \times 10^{-5}$ & $3.0 \times 10^{-3}$ & $1.3 \times 10^{-2}$ \\
.6 & $1.1 \times 10^{-5}$ & $1.8 \times 10^{-5}$ & $2.8 \times 10^{-3}$ & $1.3 \times 10^{-2}$ \\
.8 & $1.7 \times 10^{-5}$ & $3.6 \times 10^{-5}$ & $2.7 \times 10^{-3}$ & $1.3 \times 10^{-2}$ \\
1.0 & $2.3 \times 10^{-5}$ & $8.7 \times 10^{-5}$ & $2.6 \times 10^{-3}$ & $1.3 \times 10^{-2}$ \\
\hline
\end{tabular}

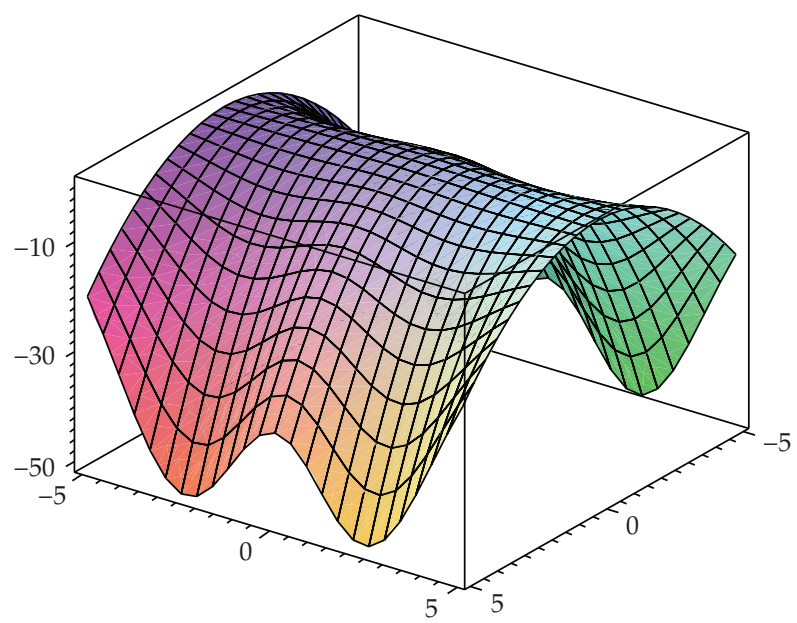

Figure 5: Depicts series solution at $\delta=.01$.

\section{Parameter Expansion Method}

Consider the following Duffing harmonic oscillator

$$
u_{t t}=-\frac{u^{3}}{1+u^{2}}, \quad u(0)=A, \quad u_{t}(0)=0
$$

We rewrite (5.1) in the form

$$
u_{t t}+o \cdot u+1 \cdot u^{2} u_{t t}+1 \cdot u^{3}=0
$$




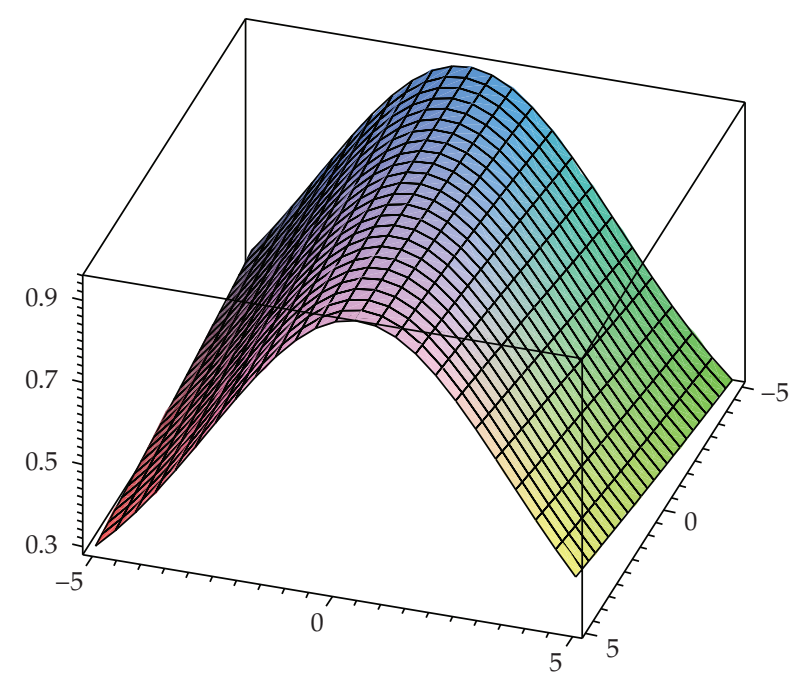

Figure 6: Depicts closed form solution at $\delta=.01$.

Assuming that the solution of (5.1) and coefficient 0,1 can be expressed as power series in $p$ as follows:

$$
\begin{gathered}
0=\omega^{2}+p a_{1}+p^{2} a_{1}+\cdots \\
1=p b_{1}+p^{2} b_{2}+\cdots
\end{gathered}
$$

Consequently, we have

$$
\begin{aligned}
& u_{0}^{\prime \prime}+\omega^{2} u_{0}=0, \quad u(0)=A, u_{t}(0)=0, \\
& u_{1}^{\prime \prime}+\omega^{2} u_{1}+a_{1} u_{0}+b_{1} u_{0}^{2} u_{0}^{\prime \prime}+b_{1} u_{0}^{3}=0, \quad u(0)=0, \quad u_{t}(0)=0 .
\end{aligned}
$$

Solving (5.5), we have $u_{0}=A \cos \omega t$, substituting $u_{0}$ in (5.6) gives

$$
\mathrm{u}_{1}^{\prime \prime}+\omega^{2} u_{1}+A \cos \omega t\left(a_{1}+\frac{3}{4} b_{1} A^{2}\left(1-\omega^{2}\right)\right)+\frac{1}{4} b_{1} A^{3}\left(1-\omega^{2}\right) \cos 3 \omega t=0
$$

Elimination of the secular term requires $a_{1}=-(3 / 4) b_{1} A^{2}\left(1-\omega^{2}\right)$. If only the first-order approximation is searched for, then $a_{1}=-\omega^{2} b_{1}$, is obtained from (5.3) and (5.4) which lead to $\omega^{2}=3 A^{2} /\left(4+3 A^{2}\right)$.

\section{Conclusion and Future Research}

In this paper, we made a detailed study of some relatively new techniques along with some of their modifications. In particular, we focused on He's VIM, HPM, MVIMS, expfunction, and expansion of parameters methods and discussed in length their respective 
applications in solving various diversified initial and boundary value problems. These proposed methods and their modifications are employed without using linearization, discretization, transformation, or restrictive assumptions, absorb the positive features of the coupled techniques and hence are very much compatible with the diversified and versatile nature of the physical problems. Moreover, the modification of VIM based upon He's polynomials (VIMHP) is easier to implement and is more user friendly as compared to the one where Adomian's polynomials (VIMAP) along with their complexities are used. It is also observed that the coupling of He's or Adomian's polynomials with the correction functional of VIM makes the solution procedure simpler and hence the evaluation of nonlinear term becomes easier. It may be concluded that the relatively new techniques can be treated as alternatives for solving a wide class of nonlinear problems.

We would like to mention that the techniques and ideas presented in this paper can be extended for finding the analytic solution of the obstacle, unilateral, free, moving, and contacts problems which arises in various branches of mathematical, physical, regional, medical, structure analysis, and engineering sciences. These problems can be studied in the general, natural, and unified framework of variational inequalities. In a variational inequality framework of such problems, the location of the contact area (free or moving boundary) becomes an integral part of the solution and no special techniques are needed to obtain it. It is well-known that if the obstacle is known then the variational inequalities can be characterized by system of variational equations. Momani et al. [101] have used Admonian decomposition technique to solve the system of fourth-order obstacle boundary value problems. This area of research is not yet developed and offers a wealth of new opportunities for further research. It is our hope that this brief introduction may inspire and motive the readers to discover new, innovative, and novel applications of these new techniques, which we have discussed in this paper. For the applications, physical formulation, numerical methods and other applications of the variational inequalities, see, Noor [102-109], Noor et al [110] and the references therein.

\section{Acknowledgments}

The authors are highly grateful to both the referees and Prof. Dr Ji-Huan He for their very constructive comments. The authors would like to thank Dr S. M. Junaid Zaidi, Rector CIIT for providing excellent research environment and facilities. The first author is also grateful Brig (R) Qamar Zaman, Vice Chancellor, HITEC University, for the provision of very conducive environs for research.

\section{References}

[1] S. Abbasbandy, "A new application of He's variational iteration method for quadratic Riccati differential equation by using Adomian's polynomials," Journal of Computational and Applied Mathematics, vol. 207, no. 1, pp. 59-63, 2007.

[2] S. Abbasbandy, "Numerical solution of non-linear Klein-Gordon equations by variational iteration method," International Journal for Numerical Methods in Engineering, vol. 70, no. 7, pp. 876-881, 2007.

[3] M. A. Abdou and A. A. Soliman, "Variational iteration method for solving Burger's and coupled Burger's equations," Journal of Computational and Applied Mathematics, vol. 181, no. 2, pp. 245-251, 2005.

[4] M. A. Abdou and A. A. Soliman, "New applications of variational iteration method," Physica D, vol. 211, no. 1-2, pp. 1-8, 2005.

[5] T. A. Abassy, M. A. El-Tawil, and H. El Zoheiry, "Solving nonlinear partial differential equations using the modified variational iteration Padé technique," Journal of Computational and Applied Mathematics, vol. 207, no. 1, pp. 73-91, 2007. 
[6] B. Batiha, M. S. M. Noorani, and I. Hashim, "Variational iteration method for solving multispecies Lotka-Volterra equations," Computers E Mathematics with Applications, vol. 54, no. 7-8, pp. 903-909, 2007.

[7] J. Biazar and H. Ghazvini, "He's variational iteration method for fourth-order parabolic equations," Computers \& Mathematics with Applications, vol. 54, no. 7-8, pp. 1047-1054, 2007.

[8] G. Adomian, "Solution of the Thomas-Fermi equation," Applied Mathematics Letters, vol. 11, no. 3, pp. 131-133, 1998.

[9] C. Y. Chan and Y. C. Hon, "A constructive solution for a generalized Thomas-Fermi theory of ionized atoms," Quarterly of Applied Mathematics, vol. 45, no. 3, pp. 591-599, 1987.

[10] A. Cedillo, "A perturbative approach to the Thomas-Fermi equation in terms of the density," Journal of Mathematical Physics, vol. 34, no. 7, pp. 2713-2717, 1993.

[11] D. D. Ganji, H. Tari, and M. B. Jooybari, "Variational iteration method and homotopy perturbation method for nonlinear evolution equations," Computers $\mathcal{E}$ Mathematics with Applications, vol. 54, no. 7-8, pp. 1018-1027, 2007.

[12] S. A. El-Wakil, M. A. Madkour, and M. A. Abdou, "Application of Exp-function method for nonlinear evolution equations with variable coefficients," Physics Letters A, vol. 369, pp. 62-69, 2007.

[13] A. Ghorbani and J. S. Nadjafi, "He's homotopy perturbation method for calculating adomian polynomials," International Journal of Nonlinear Sciences and Numerical Simulation, vol. 8, no. 2, pp. 229-232, 2007.

[14] A. Ghorbani, "Beyond adomian's polynomials: He polynomials," Chaos, Solitons E Fractals, vol. 39, no. 3, pp. 1486-1492, 2009.

[15] A. Golbabai and M. Javidi, “A variational iteration method for solving parabolic partial differential equations," Computers \& Mathematics with Applications, vol. 54, no. 7-8, pp. 987-992, 2007.

[16] J.-H. He, "Variational approach to the Thomas-Fermi equation," Applied Mathematics and Computation, vol. 143, no. 2-3, pp. 533-535, 2003.

[17] J. H. He, "An elementary introduction of recently developed asymptotic methods and nanomechanics in textile engineering," International Journal of Modern Physics B, vol. 22, no. 21, pp. 3487-4578, 2008.

[18] J.-H. He, "Some asymptotic methods for strongly nonlinear equations," International Journal of Modern Physics B, vol. 20, no. 10, pp. 1141-1199, 2006

[19] J.-H. He, "Variational iteration method-a kind of non-linear analytical technique: some examples," International Journal of Non-Linear Mechanics, vol. 34, no. 4, pp. 699-708, 1999.

[20] J.-H. He, "Variational iteration method for autonomous ordinary differential systems," Applied Mathematics and Computation, vol. 114, no. 2-3, pp. 115-123, 2000.

[21] J.-H. He and X.-H. Wu, “Construction of solitary solution and compacton-like solution by variational iteration method," Chaos, Solitons E Fractals, vol. 29, no. 1, pp. 108-113, 2006.

[22] J.-H. He, "Variational iteration method-some recent results and new interpretations," Journal of Computational and Applied Mathematics, vol. 207, no. 1, pp. 3-17, 2007.

[23] J.-H. He and X.-H. Wu, "Variational iteration method: new development and applications," Computers $\mathcal{E}$ Mathematics with Applications, vol. 54, no. 7-8, pp. 881-894, 2007.

[24] J.-H. He, "The variational iteration method for eighth-order initial-boundary value problems," Physica Scripta, vol. 76, no. 6, pp. 680-682, 2007.

[25] J.-H. He, "Bookkeeping parameter in perturbation methods," International Journal of Nonlinear Sciences and Numerical Simulation, vol. 2, no. 3, pp. 257-264, 2001.

[26] J.-H. He, "Iteration perturbation method for strongly nonlinear oscillations," Journal of Vibration and Control, vol. 7, no. 5, pp. 631-642, 2001.

[27] J.-H. He, "A new perturbation technique which is also valid for large parameters," Journal of Sound and Vibration, vol. 229, no. 5, pp. 1257-1263, 2000.

[28] J.-H. He, "Recent development of the homotopy perturbation method," Topological Methods in Nonlinear Analysis, vol. 31, no. 2, pp. 205-209, 2008.

[29] J.-H. He, "Homotopy perturbation technique," Computer Methods in Applied Mechanics and Engineering, vol. 178, no. 3-4, pp. 257-262, 1999.

[30] J.-H. He, “Homotopy perturbation method for solving boundary value problems," Physics Letters A, vol. 350, no. 1-2, pp. 87-88, 2006.

[31] J.-H. He, "Comparison of homotopy perturbation method and homotopy analysis method," Applied Mathematics and Computation, vol. 156, no. 2, pp. 527-539, 2004. 
[32] J.-H. He, "Homotopy perturbation method for bifurcation of nonlinear problems," International Journal of Nonlinear Sciences and Numerical Simulation, vol. 6, no. 2, pp. 207-208, 2005.

[33] J.-H. He, "The homotopy perturbation method nonlinear oscillators with discontinuities," Applied Mathematics and Computation, vol. 151, no. 1, pp. 287-292, 2004.

[34] J.-H. He, "A coupling method of a homotopy technique and a perturbation technique for non-linear problems," International Journal of Non-Linear Mechanics, vol. 35, no. 1, pp. 37-43, 2000.

[35] J.-H. He, "Homotopy perturbation method: a new nonlinear analytical technique," Applied Mathematics and Computation, vol. 135, no. 1, pp. 73-79, 2003.

[36] J.-H. He, "Application of homotopy perturbation method to nonlinear wave equations," Chaos, Solitons \& Fractals, vol. 26, no. 3, pp. 695-700, 2005.

[37] J.-H. He, "New interpretations of homotopy perturbation method," International Journal of Modern Physics B, vol. 20, no. 18, pp. 2561-2568, 2006.

[38] J.-H. He, "An elementary introduction to the homotopy perturbation method," Computers $\mathcal{E}$ Mathematics with Applications, vol. 57, no. 3, pp. 410-412, 2009.

[39] J.-H. He and X.-H. Wu, "Exp-function method for nonlinear wave equations," Chaos, Solitons $\mathcal{E}$ Fractals, vol. 30, no. 3, pp. 700-708, 2006.

[40] J.-H. He and M. A. Abdou, "New periodic solutions for nonlinear evolution equations using Expfunction method," Chaos, Solitons E Fractals, vol. 34, no. 5, pp. 1421-1429, 2007.

[41] Y. C. Hon, "A decomposition method for the Thomas-Fermi equation," Southeast Asian Bulletin of Mathematics, vol. 20, no. 3, pp. 55-58, 1996.

[42] D. Kaya, "An explicit and numerical solutions of some fifth-order KdV equation by decomposition method," Applied Mathematics and Computation, vol. 144, no. 2-3, pp. 353-363, 2003.

[43] D. Kaya, "On the solution of a Korteweg-de Vries like equation by the decomposition method," International Journal of Computer Mathematics, vol. 72, no. 4, pp. 531-539, 1999.

[44] J. Lu, "Variational iteration method for solving two-point boundary value problems," Journal of Computational and Applied Mathematics, vol. 207, no. 1, pp. 92-95, 2007.

[45] W. X. Ma and D. T. Zhou, "Explicit exact solution of a generalized KdV equation," Acta Mathematica Scientia, vol. 17, pp. 168-174, 1997.

[46] W.-X. Ma and Y. You, "Solving the Korteweg-de Vries equation by its bilinear form: wronskian solutions," Transactions of the American Mathematical Society, vol. 357, no. 5, pp. 1753-1778, 2005.

[47] W. X. Ma and Y. You, "Rational solutions of the Toda lattice equation in Casoratian form," Chaos, Solitons E Fractals, vol. 22, no. 2, pp. 395-406, 2004.

[48] W.-X. Ma, H. Wu, and J. He, "Partial differential equations possessing Frobenius integrable decompositions," Physics Letters A, vol. 364, no. 1, pp. 29-32, 2007.

[49] R. Mokhtari, "Variational iteration method for solving nonlinear differential-difference equations," International Journal of Nonlinear Sciences and Numerical Simulation, vol. 9, no. 1, pp. 19-23, 2008.

[50] S. Momani and S. Abuasad, "Application of He's variational iteration method to Helmholtz equation," Chaos, Solitons E Fractals, vol. 27, no. 5, pp. 1119-1123, 2006.

[51] S. T. Mohyud-Din, M. A. Noor, and K. I. Noor, "Travelling wave solutions of seventh-order generalized $\mathrm{KdV}$ equations using He's polynomials," International Journal of Nonlinear Sciences and Numerical Simulation, vol. 10, no. 2, pp. 223-229, 2009.

[52] S. T. Mohyud-Din, M. A. Noor, and K. I. Noor, "Parameter-expansion techniques for strongly nonlinear oscillators," International Journal of Nonlinear Sciences and Numerical Simulation, vol. 10, no. 5, pp. 581-583, 2009.

[53] S. T. Mohyud-Din, M. A. Noor, and A. Waheed, "Exp-function method for generalized traveling solutions of good Boussinesq equations," Journal of Applied Mathematics and Computing, vol. 30, no. 1-2, pp. 439-445, 2009.

[54] S. T. Mohyud-Din, M. A. Noor, K. I. Noor, and A. Waheed, "Exp-function method for generalized travelling solutions of Lax equations," to appear in International Journal of Modern Physics B.

[55] S. T. Mohyud-Din and M. A. Noor, "Homotopy perturbation method for solving fourth-order boundary value problems," Mathematical Problems in Engineering, vol. 2007, Article ID 98602, 15 pages, 2007.

[56] S. T. Mohyud-Din and M. A. Noor, "Homotopy perturbation method for solving partial differential equations," Zeitschrift für Naturforschung A, vol. 64a, pp. 157-171, 2009.

[57] S. T. Mohyud-Din and M. A. Noor, "Homotopy perturbation method and Padé approximants for solving Flierl-Petviashivili equation," Applications and Applied Mathematics, vol. 3, no. 2, pp. 224-234, 2008. 
[58] S. T. Mohyud-Din, M. A. Noor, and K. I. Noor, "Exp-function method for travelling wave solutions ZK-MEW equation," to appear in International Journal of Modern Physics B.

[59] S. T. Mohyud-Din, M. A. Noor, and K. I. Noor, "Exp-function method for solving nonlinear higherorder boundary value problems," to appear in Bulletin of the Institute of Academia Sinica.

[60] S. T. Mohyud-Din and M. A. Noor, "Modified variational iteration method for solving Fisher's equations," Journal of Applied Mathematics and Computing, pp. 1-14, 2008.

[61] M. A. Noor and S. T. Mohyud-Din, "Solution of singular and nonsingular initial and boundary value problems by modified variational iteration method," Mathematical Problems in Engineering, vol. 2008, Article ID 917407, 23 pages, 2008.

[62] S. T. Mohyud-Din and M. A. Noor, "Solving Schrödinger equations by modified variational iteration method," World Applied Sciences Journal, vol. 5, no. 3, pp. 352-357, 2008.

[63] S. T. Mohyud-Din and M. A. Noor, "Variational iteration method for solving discrete KdV equation," to appear in Bulletin of the Institute of Academia Sinica.

[64] S. T. Mohyud-Din, M. A. Noor, and K. I. Noor, "Modified variational iteration method for solving Sine Gordon equations," World Applied Sciences Journal, vol. 5, no. 3, pp. 352-357, 2008.

[65] M. A. Noor and S. T. Mohyud-Din, "Homotopy perturbation method for nonlinear higher-order boundary value problems," International Journal of Nonlinear Sciences and Numerical Simulation, vol. 9, no. 4, pp. 395-408, 2008.

[66] M. A. Noor and S. T. Mohyud-Din, “Variational iteration method for solving higher-order nonlinear boundary value problems using He's polynomials," International Journal of Nonlinear Sciences and Numerical Simulation, vol. 9, no. 2, pp. 141-157, 2008.

[67] M. A. Noor and S. T. Mohyud-Din, "Modified variational iteration method for heat and wave-like equations," Acta Applicandae Mathematicae, vol. 104, no. 3, pp. 257-269, 2008.

[68] M. A. Noor and S. T. Mohyud-Din, "Modified variational iteration method for solving Helmholtz equations," Computational Mathematics and Modeling, vol. 20, no. 1, pp. 40-50, 2009.

[69] M. A. Noor and S. T. Mohyud-Din, "Variational iteration method for fifth-order boundary value problems using He's polynomials," Mathematical Problems in Engineering, vol. 2008, Article ID 954794, 12 pages, 2008.

[70] M. A. Noor and S. T. Mohyud-Din, "Solution of singular and nonsingular initial and boundary value problems by modified variational iteration method," Mathematical Problems in Engineering, vol. 2008, Article ID 917407, 23 pages, 2008.

[71] M. A. Noor and S. T. Mohyud-Din, "Solution of twelfth-order boundary value problems by variational iteration technique," Journal of Applied Mathematics and Computing, vol. 28, no. 1-2, pp. 123-131, 2008.

[72] M. A. Noor and S. T. Mohyud-Din, "Modified variational iteration method for solving fourth-order boundary value problems," Journal of Applied Mathematics and Computing, vol. 29, no. 1-2, pp. 81-94, 2009.

[73] M. A. Noor and S. T. Mohyud-Din, "Modified variational iteration method for Goursat and Laplace problems," World Applied Sciences Journal, vol. 4, no. 4, pp. 487-498, 2008.

[74] M. A. Noor and S. T. Mohyud-Din, "Variational iteration decomposition method for solving eighthorder boundary value problems," Differential Equations and Nonlinear Mechanics, vol. 2007, Article ID 19529, 16 pages, 2007.

[75] M. A. Noor, S. T. Mohyud-Din, and A. Waheed, "Exp-function method for solving KuramotoSivashinsky and Boussinesq equations," Journal of Applied Mathematics and Computing, vol. 29, no. 1-2, pp. 1-13, 2009

[76] M. A. Noor, S. T. Mohyud-Din, and A. Waheed, "Exp-function method for generalized traveling solutions of master partial differential equation," Acta Applicandae Mathematicae, vol. 104, no. 2, pp. 131-137, 2008.

[77] M. Rafei and H. Daniali, "Application of the variational iteration method to the Whitham-BroerKaup equations," Computers $\mathcal{E}$ Mathematics with Applications, vol. 54, no. 7-8, pp. 1079-1085, 2007.

[78] N. H. Sweilam, "Harmonic wave generation in non linear thermoelasticity by variational iteration method and Adomian's method," Journal of Computational and Applied Mathematics, vol. 207, no. 1, pp. 64-72, 2007.

[79] N. H. Sweilam, "Fourth order integro-differential equations using variational iteration method," Computers \& Mathematics with Applications, vol. 54, no. 7-8, pp. 1086-1091, 2007.

[80] M. Tatari and M. Dehghan, "On the convergence of He's variational iteration method," Journal of Computational and Applied Mathematics, vol. 207, no. 1, pp. 121-128, 2007. 
[81] A.-M. Wazwaz, "The modified decomposition method and Padé approximants for solving the Thomas-Fermi equation," Applied Mathematics and Computation, vol. 105, no. 1, pp. 11-19, 1999.

[82] A.-M. Wazwaz, "Analytic treatment for variable coefficient fourth-order parabolic partial differential equations," Applied Mathematics and Computation, vol. 123, no. 2, pp. 219-227, 2001.

[83] A.-M. Wazwaz, "The decomposition method for approximate solution of the Goursat problem," Applied Mathematics and Computation, vol. 69, no. 2-3, pp. 299-311, 1995.

[84] X.-H. Wu and J.-H. He, "Solitary solutions, periodic solutions and compacton-like solutions using the Exp-function method," Computers \& Mathematics with Applications, vol. 54, no. 7-8, pp. 966-986, 2007.

[85] X.-H. Wu and J.-H. He, "Solitary solutions, periodic solutions and compacton-like solutions using the Exp-function method," Computers E Mathematics with Applications, vol. 54, no. 7-8, pp. 966-986, 2007.

[86] X.-H. Wu and J.-H. He, "EXP-function method and its application to nonlinear equations," Chaos, Solitons \& Fractals, vol. 38, no. 3, pp. 903-910, 2008.

[87] X.-H. Wu and J.-H. He, "Solitary solutions, periodic solutions and compacton-like solutions using the Exp-function method," Computers \& Mathematics with Applications, vol. 54, no. 7-8, pp. 966-986, 2007.

[88] L. Xu, "The variational iteration method for fourth order boundary value problems," Chaos, Solitons E Fractals, vol. 39, no. 3, pp. 1386-1394, 2009.

[89] L. Xu, "He's homotopy perturbation method for a boundary layer equation in unbounded domain," Computers \& Mathematics with Applications, vol. 54, no. 7-8, pp. 1067-1070, 2007.

[90] L. Xu, "Variational iteration method for solving integral equations," Computers E Mathematics with Applications, vol. 54, no. 7-8, pp. 1071-1078, 2007.

[91] L. Xu, "He's parameter-expanding methods for strongly nonlinear oscillators," Journal of Computational and Applied Mathematics, vol. 207, no. 1, pp. 148-154, 2007.

[92] L. Xu, "Application of He's parameter-expansion method to an oscillation of a mass attached to a stretched elastic wire," Physics Letters A, vol. 368, no. 3-4, pp. 259-262, 2007.

[93] L. Xu, "Determination of limit cycle by He's parameter-expanding method for strongly nonlinear oscillators," Journal of Sound and Vibration, vol. 302, no. 1-2, pp. 178-184, 2007.

[94] E. Yusufoğlu, "New soliton solutions for the MBBN equations using exp-function method," Physics Letters A, vol. 372, no. 4, pp. 442-446, 2008.

[95] A. Ylldirım, "Applying He's variational iteration method for solving differential-difference equation," Mathematical Problems in Engineering, vol. 2008, Article ID 869614, 7 pages, 2008.

[96] L.-N. Zhang and L. Xu, "Determination of the limit cycle by He's parameter-expansion for oscillators in a $\mathrm{u}(3) /(1+\mathrm{u}(2))$ potential," Zeitschrift für Naturforschung A, vol. 62, no. 7-8, pp. 396-398, 2007.

[97] X.-W. Zhou, Y.-X. Wen, and J.-H. He, "Exp-function method to solve the nonlinear dispersive K(m, n) equations," International Journal of Nonlinear Sciences and Numerical Simulation, vol. 9, no. 3, pp. 301-306, 2008.

[98] S. Zhang, "Application of Exp-function method to high-dimensional nonlinear evolution equation," Chaos, Solitons \& Fractals, vol. 365, pp. 448-455, 2007.

[99] S.-D. Zhu, "Exp-function method for the Hybrid-Lattice system," International Journal of Nonlinear Sciences and Numerical Simulation, vol. 8, no. 3, pp. 461-464, 2007.

[100] S.-D. Zhu, "Exp-function method for the discrete mKdV lattice," International Journal of Nonlinear Sciences and Numerical Simulation, vol. 8, no. 3, pp. 465-468, 2007.

[101] S. Momani, K. Moadi, and M. A. Noor, "Decomposition method for solving a system of fourth-order obstacle boundary value problems," Applied Mathematics and Computation, vol. 175, no. 2, pp. $923-$ 931, 2006.

[102] M. A. Noor, On variational inequalities, Ph.D. thesis, Brunel University, London, UK, 1975.

[103] M. A. Noor, "Some recent advances in variational inequalities-I: basic concepts," New Zealand Journal of Mathematics, vol. 26, no. 1, pp. 53-80, 1997.

[104] M. A. Noor, "Some recent advances in variational inequalities-II: other concepts," New Zealand Journal of Mathematics, vol. 26, no. 2, pp. 229-255, 1997.

[105] M. A. Noor, "Some developments in general variational inequalities," Applied Mathematics and Computation, vol. 152, no. 1, pp. 199-277, 2004.

[106] M. A. Noor, “General variational inequalities,” Applied Mathematics Letters, vol. 1, no. 2, pp. 119-122, 1988.

[107] M. A. Noor, "Fundamentals of mixed quasi variational inequalities," International Journal of Pure and Applied Mathematics, vol. 15, no. 2, pp. 137-258, 2004. 
[108] M. A. Noor, "Hemivariational-like inequalities," Journal of Computational and Applied Mathematics, vol. 182, no. 2, pp. 316-326, 2005.

[109] M. A. Noor, "New approximation schemes for general variational inequalities," Journal of Mathematical Analysis and Applications, vol. 251, no. 1, pp. 217-229, 2000.

[110] M. A. Noor, K. I. Noor, and Th. M. Rassias, "Some aspects of variational inequalities," Journal of Computational and Applied Mathematics, vol. 47, no. 3, pp. 285-312, 1993. 


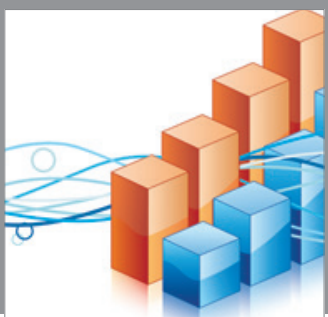

Advances in

Operations Research

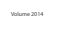

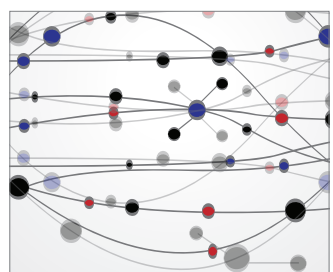

\section{The Scientific} World Journal
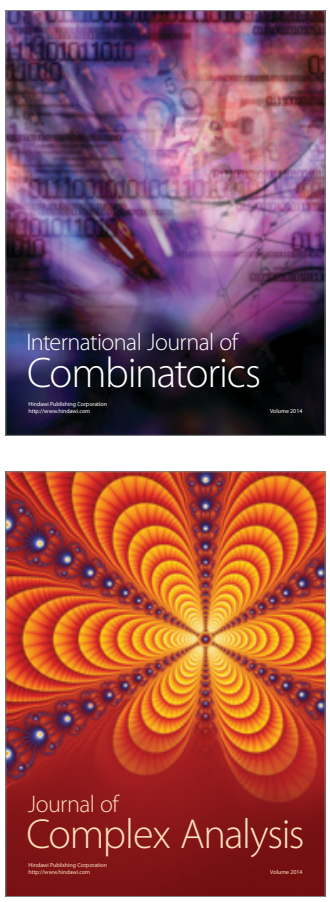

International Journal of

Mathematics and

Mathematical

Sciences
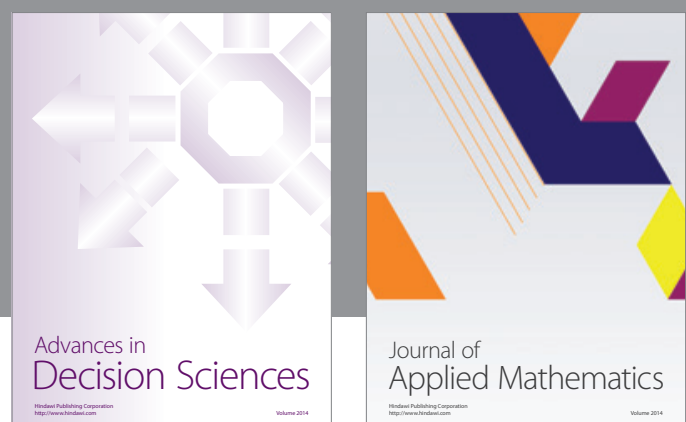

Journal of

Applied Mathematics
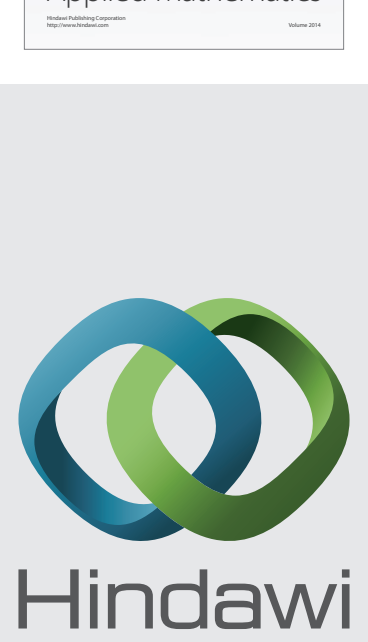

Submit your manuscripts at http://www.hindawi.com
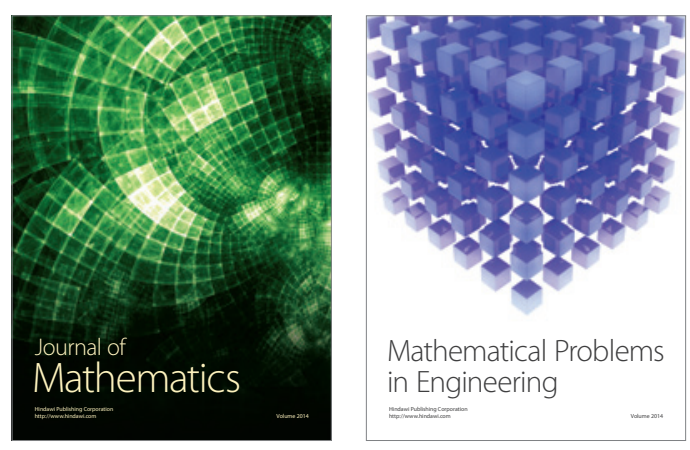

Mathematical Problems in Engineering
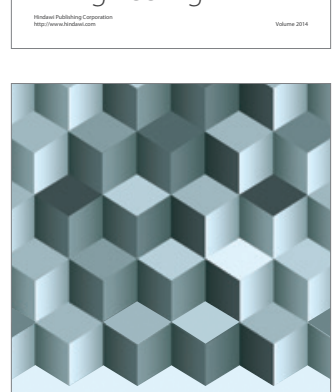

Journal of

Function Spaces
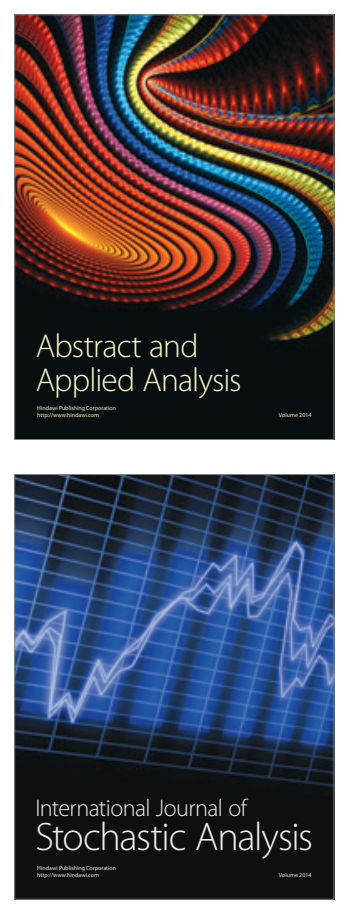

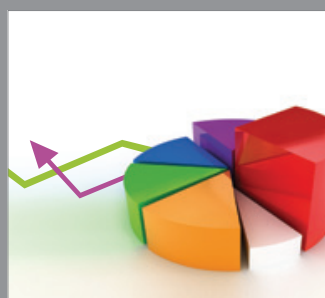

ournal of

Probability and Statistics

Promensencen
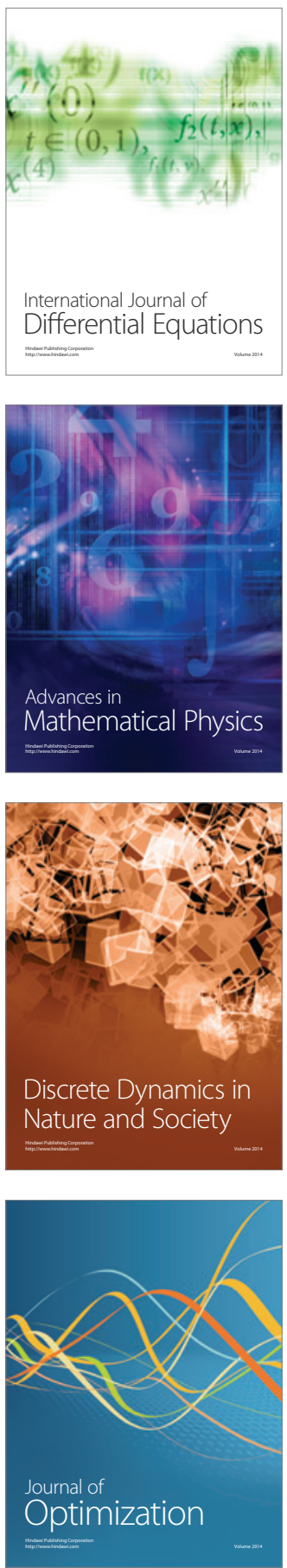